\title{
Increasing Complexity
}

Dear Reader,

NVH has always been an important indicator of a vehicle's engineering and quality, and has therefore always been the focus of developers. And of course there has always been the cry for improvements from one vehicle generation to the next, not only by applying large-scale measures, but often also very minor ones. But some of today's developers will probably mourn the passing of the good old days. They were characterized by a one-dimensional internal combustion engine and the requirements were therefore always subject to the fact that the combustion engine not only generated certain noises, but also concealed them. By contrast, the greater diversity and complexity of today's drivetrains - not least due to electric mobility - means that engineers are now facing even more diverse challenges. What measures can be taken to reduce noise that is no longer superimposed? Which measures are necessary to deal with new noises caused by the variety of drive trains? How can unavoidable noise be made as pleasant as possible? In addition, the requirements for interior acoustics will continue to increase in the future. A key role is also being played by the increasing use of voice control in the form of HMI, but also by a greater use of 'infotainment' services, as well as customer comfort requirements in the context of automated driving. The discussion concerning driving range means that lightweight design is also gaining in importance, as are new and lighter materials, for example for insulation. Engineers are addressing all of these challenges with, among other things, better measurement analyses and faster calculation methods. In cooperation with Autoneum, we have compiled some examples for you in this ATZextra.

This special issue is always published in the years between our biennial Automotive Acoustics Conference. I very much hope that we have once again succeeded in offering you numerous interesting articles to pass the time until the next conference, which will take place in Zurich (Switzerland) on 9 and 10 July 2019, by offering numerous interesting articles. With this in mind, I hope you enjoy reading our special issue.

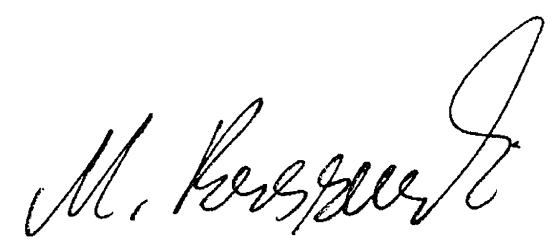

Markus Bereszewski

Head of Corporate Solutions/Editor in Chief

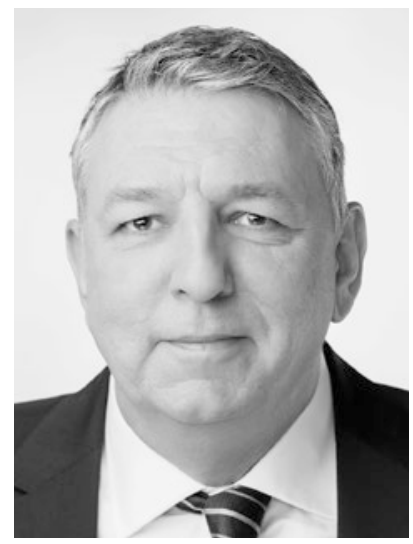

